\title{
Precordial ST Segment Depression on Admission Electrocardiogram as a Simple Noninvasive Tool for Predicting Coronary Artery Disease Complexity in Patients with Inferior Myocardial Infarction
}

\author{
Jaya Suganti, Anggia Chairuddin Lubis, Abdullah Afif Siregar, Andika Sitepu,
} Cut Aryfa Andra, Ali Nafiah Nasution, Harris Hasan

\begin{abstract}
Background: Whether a precordial ST segment depression (PSTD) is merely a benign electrical phenomena or a sign of multivessel coronary artery disease (MVCAD) in inferior myocardial infarction (MI) remains unclear. The objective of this study is to analyze the complexity of coronary artery disease (CAD) in inferior MI patients with PSTD and to investigate whether PSTD can be used as a predictor of MVCAD in inferior MI.

Methods: Patients with inferior Ml were divided into two groups based on the presence of PSTD on admission ECG and were compared based on the patient's coronary artery complexities.

Results: A total of 215 patients with inferior MI were found in this study period, with 102 patients meet the inclusion and exclusion criteria. Patients with PSTD had a higher incidence of MVCAD and SYNTAX score. Further analyzes showed PSTD on admission ECG was an independent predictor of MVCAD in inferior MI [45 (66\%) vs 23 (34\%); OR 4.097; 95\% CI I.638-I0.247; $p=0.003$ ).

Conclusion: In daily clinical practice, PSTD on admission ECG may serve as a simple noninvasive tool for predicting MVCAD or a more complex CAD in inferior MI.
\end{abstract}

(Indonesian J Cardiol. 20 I9;40:3 I2-3 I8)

Keywords: Precordial ST Segment depression, inferior myocardial infarction, SYNTAX score

Department of Cardiology and Vascular Medicine, Faculty of Medicine University of Sumatera Utara, Haji Adam Malik General Hospital, Medan, Indonesia

\section{Correspondence:}

dr. Anggia Chairuddin Lubis, Sp.JP, FIHA

Department of Cardiology and Vascular Medicine

University of Sumatera Utara - Haji Adam Malik General

Hospital

E-mail: lubis_ac@hotmail.com

\section{Introduction}

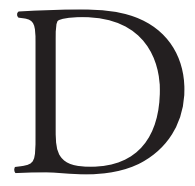

uring an episode of ST segment elevation myocardial infarction (STEMI), a persistent ST segment elevation in leads facing the infarct area were often accompanied by ST segment depression in leads remote from infarct area (noninfarct area) $)^{1}$. Approximately $60 \%$ of patients with acute inferior myocardial infarction (MI) showed an 
electrocardiographic evidence of ST segment depression in the precordial leads ${ }^{2}$. Initially, precordial ST segment depression (PSTD) in inferior MI was believed as benign electrical phenomenon with no anatomic or physiologic significance 3,4 . Contrary to this opinion, numbers of studies have shown that the presence of PSTD in inferior MI was associated with a more extensive myocardial ischemia or a larger infarcts as evidenced by higher cardiac enzymes level, more severe regional wall motion abnormalities, lower left ventricular ejection fraction (LVEF). As a result, patients with PSTD are proned to short term and long term complications compare to patients without PSTD. Thus, the presence of PSTD defines a high risk subset of patients with inferior MI due to a higher incidence of major adverse cardiovascular events (MACE) $)^{3,5-7}$.

Furthermore, it is very important to identify the etiology of PSTD in inferior MI and more spesifically the mechanism underlying this ECG finding. Two scenarios may answer this question. First, PSTD in inferior MI is caused by an occlusion of infarct related artery with larger myocardial perfusion territories ${ }^{8-10}$. As a consequence, this electrocardiographic pattern did not represent an incidental electrical event, but reflected a large mass of myocardium at risk for infarction without involvement of multivessel disease ${ }^{7}$. Therefore, this ECG pattern indicate the extension of infarction to the posterior, lateral or posterolateral segment of left ventricle during inferior MI without the involvement of other vessels except the infarct related artery (single vessel coronary artery disease) ${ }^{11,12}$. Second, the mechanism of PSTD in inferior MI is best described by using the concept of "ischemia at a distance" which suggested that an acute occlusion of the right coronary artery (RCA) may produce ischemia in the myocardium supplied by left anterior descending artery (LAD) if it has significant stenosis and depends on collateral blood flow from the RCA $^{13,14}$. Precordial ST segment depression represent a concomitant anterior ischemia due to LAD disease (multivessel coronary artery disease in inferior MI) ${ }^{15-18}$. Thus, two mechanisms with different pathophysiologic bases may produce the same ECG pattern.

Until now, the certainty of this issue is still under investigation with conflicting results. To provide an answer to this issue, the present study will focus on analyzing the extent and severity of coronary artery disease (CAD) in relation with PSTD in patients with inferior MI. Therefore, the aim of this study is to analyze the complexity of CAD in inferior MI patients with PSTD and to investigate whether PSTD in inferior MI can be used as a predictor of MVCAD.

\section{Methods}

\section{Study Design and Patients Population}

A single centre retrospective study was conducted on consecutive series of patients with inferior MI who were admitted to Emergency Unit, Department of Cardiology and Vascular Medicine, Haji Adam Malik General Hospital, Medan, Indonesia, between December 2013 and December 2016.

The diagnosis of MI was based on the 2013 ACCF/ AHA Guideline for the Management of ST-Elevation Myocardial Infarction ${ }^{19}$. Inferior MI was defined as the presence of ST segment elevation in at least two leads representing the inferior wall (II, III, aVF) ${ }^{20}$.

The inclusion criteria were as follow : (1) inferior MI patients with onset $<7$ days who underwent coronary angiography during hospitalization; (2) no history or electrocardiographic evidence of previous MI; (3) no history of revascularization : percutaneous coronary intervention (PCI) or coronary artery bypass graft $(\mathrm{CABG})$; (4) no history or clinical findings of valvular heart disease, cardiomyopathy, or other cardiac disease, (5) was not consuming digitalis preparations or other drugs known to alter the ST segment. Patients with several ECG patterns such as bundle branch block, left ventricle hypertrophy, ventricular paced rhythm, or a coronary angiographic evidence of left coronary dominance were excluded from the study. The current study sample consisted of patients with inferior MI who met the inclusion and exclusion criteria.

\section{Data Collection}

\section{Electrocardiogram Analyzes}

A standard 12-lead ECG $(25 \mathrm{~mm} / \mathrm{s}$ and $10 \mathrm{~mm} / \mathrm{mV})$ were obtained from all patients upon admission to emergency unit and the precordial ST segment were analyzed by a blinded observer who was unaware of the clinical and angiographic findings (Figure 1). The 
very beginning of the ST segment is called J-point ${ }^{21,22}$. The J-point is used to determine the magnitude of ST segment depression (Figure 2). By using handheld calliper and magnifying glass, PSTD was measured at the J-point in all precordial leads with the preceding TP segment as a preferred baseline. Precordial ST segment depression defined as horizontal or downsloping ST segment depression of $\geq 1 \mathrm{~mm}$ in at least two contiguous leads out of six precordial leads (V1-V6) in patients with inferior MI. In addition to standard 12-lead ECG, posterior $(\mathrm{V} 7, \mathrm{~V} 8, \mathrm{~V} 9)$ and lateral $(\mathrm{V} 5, \mathrm{~V} 6, \mathrm{I}, \mathrm{aVL}) \mathrm{ECG}$ of patients with PSTD were analyzed for posterolateral segments involvement.

\section{Coronary Angiogram Analyzes}

All coronary angiograms were analyzed by two experienced cardiologist blinded to the clinical data and ECG findings. In this study, the complexity (the extent and severity) of CAD was quantified with visualization and SYNTAXscores. Visualization is a method to quantify the complexity of CAD according to the number of major epicardial vessels with significant lesion. A lesion was defined as significant when it caused 50\% reduction in luminal diameter by visual assessment ${ }^{23}$. Based on this method, complexity of CAD was categorized into one vessel disease, two vessel disease, three vessel disease. Furthermore, one vessel disease was termed single vessel coronary artery disease (SVCAD), while two vessel disease and three vessel disease were termed MVCAD. SYNTAX (SYNergy between percutaneous coronary intervention with TAXus and cardiac surgery) score was used for a more precise measurement of lesion burden or complexity of CAD in patients with inferior MI. Each patient's SYNTAX score was calculated by answering a series of questions using the openly accessible web based score calculator (http://www.syntaxscore.com) ${ }^{23,24}$.

\section{Study Protocol}

We retrospectively studied patients with inferior MI who were admitted to Emergency Unit, Department of Cardiology and Vascular Medicine, Haji Adam Malik General Hospital, Medan, Indonesia, between December 2013 and December 2016. The study patients were divided into two groups based on the presence of PSTD on admission ECG and further comparisons were made to analyze their relationship to coronary complexities. The study protocol was reviewed and approved by the Health Research Ethical Committee University of Sumatera Utara in accordance with the Neuremberg Code and Declaration of Helsinki.

\section{Statistical Analysis}

Continuous variables were expressed as mean \pm standard deviation (SD), and were compared with Student's t-test or Mann Whitney $U$ test on the basis of their distribution (normal distribution was assessed with KolmogorovSmirnov test). Categorical data are presented as counts (n) and percentage (\%), and were compared with Chisquare or Fisher's exact test, as appropriate. A multiple logistic regression analysis was performed to investigate whether PSTD may serve as an independent predictor of MVCAD in inferior MI. Odds ratio (OR) and 95\% confidence interval (CI) were calculated. In addition, as the SYNTAX score was calculated independently by two cardiologist, a quantitative measure of agreement was needed. The degree of agreement between these observers was measured using the Cohen's kappa $(\kappa)$ statistic to analyze the inter-observer variability. In this study, $\mathrm{p}$ value $<0.05$ was considered statistically significant.

A total of 215 patients with inferior MI were found in this study period, with 102 patients met the inclusion and exclusion criteria and were eligible for further analyzes, whereas 113 patients were excluded due to the following reasons : 36 patients with incomplete data; 62 patients did not undergo coronary angiography, 5 patients with history of MI; 2 patients with history of PCI; 5 patients with bundle branch block; 3 patients with left ventricle hyperthrophy. Of the 102 patients with inferior MI, 55 patients (54\%) with PSTD on admission ECG, whereas 47 patients (46\%) without PSTD.

\section{Results}

\section{Baseline Demographic and Clinical Characteris- tics}

No significant differences were observed in mean age and gender between both groups, however, hypertension 
was more frequent in patients with PSTD. There were significant differences between both groups regarding the onset of MI, cardiac enzymes, mean LVEF, KILLIP class and reperfusion therapy. Patients with PSTD had an earlier onset of MI $[19 \pm 18$ hour vs $42 \pm 40$ hour, $\mathrm{p}=0.005]$, higher level of Troponin $\mathrm{T}[1,44 \mu \mathrm{g} / \mathrm{L}$ vs $1,07 \mu \mathrm{g} / \mathrm{L}, \mathrm{p}=0.014$ ], lower mean LVEF [ $48 \%$ vs $53 \%$, $\mathrm{p}=0.005]$. In addition, our results showed that KILLIP class $\geq 2$ and reperfusion therapy with fibrinolytics were more frequent in patients with PSTD than without PSTD [13 (24\%) vs 1 (2\%), p=0.002] and [22 (40\%) vs $6(13 \%), \mathrm{p}=0.002$ (Table 1 ).

\section{Coronary Angiographic Characteristics}

Both groups had comparable coronary angiography timing after admission. In respect to the numbers of involved coronary arteries, one vessel disease were more frequent in patients without PSTD than with PSTD [24 (51\%) vs 10 (18\%), $\mathrm{p}=0.001$ ], whereas three vessel disease and left main disease were more frequent in patients with PSTD than without PSTD [23 (42\%) vs $4(9 \%), \mathrm{p}=0.001$ ], [7 (13\%) vs $0(0 \%), \mathrm{p}=0.014]$. There was a significant difference between both groups regarding LAD disease, patients with PSTD have a higher incidence of LAD disease than patients without PSTD [ 41 (75\%) vs 16 (34\%); p<0.001; OR 5.674; 95\% CI 2.412-13.350). Further analyzes on LAD artery revealed that proximal segment of LAD with stenosis $\geq$ $70 \%$ were more common in patients with PSTD than without PSTD [26 (47\%) vs 8 (17\%), p=0.012] (Table 2). Of the 41 patients with PSTD and LAD disease, 19 patients $(46 \%)$ demonstrated collateral from the RCA to LAD and 22 patients (54\%) without collateral from RCA to LAD.

\section{The Complexity of Coronary Artery Disease in Patients of Inferior Myocardial Infarction With or Without Precordial ST Segment Depression}

The incidence of MVCAD was higher in patients with PSTD than patients without PSTD, [45 (66\%) vs 23 (34\%); OR 4.696; 95\% CI 1.923-11.436; $\mathrm{p}<0.001]$, conversely the incidence of SVCAD was higher in patients without PSTD than patients with PSTD [24 (71\%) vs $10(29 \%)]$. In other words, there were 10 patients with PSTD but without MVCAD and evaluation of posterior and lateral ECG of these patients revealed that 1 patients with posterolateral wall involvement, 2 patients with posterior wall involvement, 4 patient without posterolateral involvement, and 3 patients with incomplete data. Further analyzes revealed that total SYNTAX score was also higher in patients with PSTD than without PSTD [20 \pm 12 vs $10 \pm 7, \mathrm{p}<0.001]$. In the present study, the Cohen's Kappa Coefficient $(\kappa)$ for SYNTAX score was 0.848 (strong agreement) with $\mathrm{p}$ value $\mathrm{p}<0.001$.

\section{Precordial ST Segment Depression as a Predic- tor of Multivessel Coronary Artery Disease in Inferior Myocardial Infarction}

Initially, we performed univariate analyzes on several independent predictors of MVCAD. Significant variables with $\mathrm{p}$ value $<0.25$ in the univariate analyzes (hypertension, diabetes mellitus, heart failure on admission, ST segment elevation $\geq 1 \mathrm{~mm}$ in lead aVR) were included in multivariate analyzes with multiple logistic regression model. At the final step of multiple logistic regression analyzes, we found that PSTD on admission ECG (OR 4.097; 95\% CI 1.638-10.247; $\mathrm{p}=0.003$ ) and hypertension (OR 3.231; 95\% CI 1.135-9.196; $\mathrm{p}=0.028$ ) were independent predictors of MVCAD in inferior MI (Table 3).

\section{Discussion}

In this study, the proportion of inferior MI patients with PSTD was 54\%, and this number was almost similar as reported by Sato et al with 60\%2. Previous studies demonstrated that patients with PSTD had more complex CAD than patients without PSTD ${ }^{16}$. We thought it was probably due to a higher prevalence of CAD risk factors, however, we did not find any statistically significant differences regarding these risk factors between both groups, except for hypertension. Similarly, Nour et al. found no differences in CAD risk factors between patients with and without PSTD ${ }^{16}$. Consistent with the findings of previous studies, our results showed that the presence of PSTD in inferior MI was associated with a more extensive myocardial ischemia or a larger infarcts as evidenced by a higher level of cardiac enzymes, a lower mean LVEF, and therefore, 
the rate of MACE (acute heart failure) as represented by KILLIP class $\geq 2$ was also higher ${ }^{3,5-7}$.

We found statistically differences between the two groups concerning the onset of MI. More than half patients with PSTD had an onset of MI $\leq 12$ hours, conversely, patients without PSTD were more likely to have an onset of MI $>12$ hours. This result might explain why reperfusion therapy are more common in patients with PSTD than without PSTD. It also provides an answer regarding numbers of patients with MVCAD but were not presented as PSTD because this ECG pattern may disappeared as the onset of $\mathrm{MI}>12$ hours. Haraphongse et al. found that earliest recorded ECG is the most valuable tool in identifying high risk subset of patients because PSTD tends to disappear within 24 hours17. In addition, the presence of persistent PSTD was highly predictive of left coronary artery (LCA) disease involvement, as concluded by Kouvaras et al.18. Therefore, we suggest that persistent PSTD is a more specific marker for identifying MVCAD in inferior $\mathrm{MI}$ and further research are required to determine the association between persistent PSTD and MVCAD.

Previous studies have suggested that PSTD may represent a concomitant anterior wall ischemia due to left anterior descending (LAD) disease (MVCAD in inferior MI)15-18. Consistent with this finding, our results showed that LAD disease, more spesifically the proximal segment of LAD with occlusive lesion (100\%) or nonocclusive lesion $(\geq 70 \%)$ were more frequent in patients with PSTD than without PSTD. Eventhough this study does not provide a direct measurement or proves the existence of anterior myocardial ischemia, but these data showed that most patients with PSTD have a high grade LAD disease. We are also interested in investigating the mechanism of anterior myocardial ischemia in the context of LAD disease and based on previous literature, two possibility may explain this findings : (1) "ischemia at a distance" (2) increasing of regional myocardial oxygen demand in the anterior wall as a respond to inferior infarction ${ }^{13,14}$. Of the 41 patients with PSTD and LAD disease, 19 patients demonstrated collateral from RCA to LAD, whereas the remaining 22 patients without angiographic evidence of collateral flow. In this latter group, the second possibility may provide an explanation. We also noticed 14 patients with PSTD but without LAD disease, and two possibilities may describe this findings: (1) PSTD represent an extension of infarction to posterior, lateral or posterolateral segment of left ventricle during an inferior MI (2) spasm of LAD artery ${ }^{12,14}$.

Patients with PSTD had a higher incidence of MVCAD than patients without PSTD [45 (66\%) vs 23 (34\%); OR 4.696; 95\% CI 1.923-11.463; $\mathrm{p}<0.001]$ and our results support previous studies 10,16. Interestingly, 23 patients without PSTD had MVCAD and three possibilities may explain this findings : (1) The onset of MI was $>12$ hours and persistent PSTD did not occur (2) MVCAD without LAD involvement (3) MVCAD with LAD involvement, but the lesion are not complex and located at a more distal segment of LAD, thus it will not give significant ischemic burden to induce a PSTD. Of the 23 patients with MVCAD but without PSTD, 16 patients (70\%) have an onset of MI > 12 hours, whereas 7 patients (30\%) have an onset of $\mathrm{MI} \leq$ 12 hours. We performed a further analyzes on these 7 patients and the results were : 2 patients without LAD disease $(\mathrm{RCA}+\mathrm{LCx}), 1$ patients with lesion (stenosis $50 \%$ ) at mid segment of LAD (SYNTAX score 8), 1 patients with lesion (stenosis $80 \%$ ) at distal segment of LAD (SYNTAX score 5), however, there are 3 patients with complex lesion that involve the LAD (SYNTAX score $31,25,20)$. A deeper analyzes was needed to determine other factors that may contribute or prevent the formation of PSTD in these patients.

Brymer et al. postulated that PSTD usually occurred after occlusion of artery with an unusually large anatomic distribution, it reflected a large mass of myocardium at risk for infarction without multivessel disease ${ }^{7}$. Thus, PSTD indicate the extension of infarction to posterior, lateral or posterolateral segment of left ventricle during inferior MI. In agreement with this findings, our results showed 10 (39\%) patients with PSTD but without MVCAD. Of the 10 patients, 3 patients demonstrated posterior and lateral wall involvement. We concluded that PSTD may also represent an extension of inferior infarction to the posterolateral segment, however, these findings were insignificant compared to numbers of patients with PSTD who has MVCAD.

To the best of our knowledge, this is the first study investigating the complexity of CAD in patients of inferior MI with PSTD using SYNTAX score and assessing whether PSTD on admission ECG can be used as a predictor of MVCAD in inferior MI. Our results showed statistically significant differences between both 
groups regarding total SYNTAX score. Patients with PSTD had more complex CAD than patients without PSTD, as represented by higher mean total SYNTAX score $[20 \pm 12$ vs $10 \pm 7, \mathrm{p}<0.001]$. In contrast to our expectation, the mean total SYNTAX score of patients with PSTD was still consider as low lesion complexity (SYNTAX score $\leq 22$ ) and the reason for this findings are patients with PSTD received reperfusion therapy (fibrinolytics) more frequent than patients without PSTD and this intervention will definitely affect the total SYNTAX score. Finally, multiple logistic regression analyzes revealed that inferior MI patients with PSTD on admission ECG had a 4.1-fold increased risk of MVCAD than patients without PSTD (OR 4.097; 95\% CI 1.638-10.247; $\mathrm{p}=0.003$ ).

\section{Conclusion}

The presence of precordial ST segment depression on admission ECG in inferior MI defines a subset of patients with a higher incidence of MVCAD. In clinical practice, it may serves as a simple noninvasive tool for predicting MVCAD or a more complex CAD in patients with inferior MI.

\section{Study Limitations}

This study was limited by its retrospective design and the relatively small sample size compared to previous studies. Differences in MI onset, lytics therapy and higher risk patients in PSTD group will affect the SYNTAX score.

\section{Ethical Clearance}

This research was approved by the Health Research Ethical Committee, Faculty of Medicine University of Sumatera Utara. Ethical Clearance No.298/TGL/KEPK FK USU-RSUP HAM/2017.

\section{Publication Agreement}

The manuscript has been approved by all named authors. The authors give permission to Indonesian Journal of Cardiology to publish this article if this article is accepted.

\section{Conflict Of Interest}

The authors have no conflict of interest to declare.

\section{Funding Sources}

None.

\section{List of Abbreviations}

CAD : Coronary artery disease

DSST : Depresi segmen ST

IMAEST : Infark miokard akut elevasi segmen ST

MACE : Major adverse cardiovascular event

MI : Myocardial Infarction

MVCAD : Multivessel coronary artery disease

PSTD : Precordial ST segment depression

SVCAD: Single vessel coronary artery disease

SYNTAX : Synergy between percutaneous coronary

intervention with taxus and cardiac surgery

\section{References}

1. Ferguson DW, Pandian N, Kioschos JM, Marcus ML, White CW. Angiographic evidence that reciprocal ST-segment depression during acute myocardial infarction does not indicate remote ischemia: Analysis of 23 patients. American Journal of Cardiology. 1984;53(1):55-62.

2. Sato H, Kodama K, Masuyama T, et al. Right coronary artery occlusion: its role in the mechanism of precordial st segment depression. Journal of the American College of Cardiology. 1989;14(2):297302.

3. Berger PB, Ryan TJ. Inferior myocardial infarction. High-risk subgroups. Circulation. 1990;81(2):401411.

4. Mirvis DM. Physiologic bases for anterior ST segment depression in patients with acute inferior wall myocardial infarction. American Heart Journal. 1988;116(5):1308-1322.

5. Pichler M, Shah PK, Peter T, et al. Wall motion abnormalities and electrocardiographic changes in acute transmural myocardial infarction: Implications of reciprocal ST segment depression. American Heart Journal. 1983;106(5):1003-1009.

6. Herlitz J, Hjalmarson AA. Occurrence of anterior 
st depression in inferior myocardial infarction and relation to clinical outcome. Clinical Cardiology. 1987;10(9):529-534.

7. Gelman JS, Saltups A. Precordial ST segment depression in patients with inferior myocardial infarction: clinical implications. Heart. 1982;48(6):560565.

8. Brymer JF, Khaja F, Marzilli M, Goldstein S, Alban J. "Ischemia at a distance" during intermittent coronary artery occlusion: A coronary anatomic explanation. Journal of the American College of Cardiology. 1985;6(1):41-45.

9. Cannon RO. Ischemia at a distance-So close yet so far. Journal of the American College of Cardiology. 1985;6(1):46-48.

10. Roubin GS, Shen WF, Nicholson M, Dunn RF, David TK, Phillip JH. Anterolateral ST segment depression in acute inferior myocardial infarction: Angiographic and clinical implications. American Heart Journal. 1984;107(6):1177-1182.

11. Gibson RS, Crampton RS, Watson DD, et al. Precordial ST-segment depression during acute inferior myocardial infarction: clinical, scintigraphic and angiographic correlations. Circulation. 1982;66(4):732-741.

12. Goldberg HL, Borer JS, Jacobstein JG, Kluger J, Scheidt SS, Alonso DR. Anterior S-T segment depression in acute inferior myocardial infarction: Indicator of posterolateral infarction. American Journal of Cardiology. 1981;48(6):1009-1015.

13. Blumgart HL, Schlesinger MJ, Davis D. Studies on the relation of the clinical manifestations of angina pectoris, coronary thrombosis, and myocardial infarction to the pathologic findings: With particular reference to the significance of the collateral circulation. American Heart Journal. 1940;19(1):1-91.

14. Schuster EH, Bulkley BH. Ischemia at a distance after acute myocardial infarction: a cause of early postinfarction angina. Circulation. 1980;62(3):509515.

15. Salcedo JR, Baird MG, Chambers RJ, Beanlands DS. Significance of reciprocal S-T segment depression in anterior precordial leads in acute inferior myocardial infarction: Concomitant left anterior descending coronary artery disease? American Journal of Cardiology. 1981;48(6):1003-1008.

16. Nour MK. Significance of reciprocal ST segment depression in ST elevation myocardial infarction. The Egyptian Journal of Critical Care Medicine. 2017;5(1):23-27.

17. Haraphongse M, Jugdutt BI, Rossall RE. Significance of precordial ST-segment depression in acute transmural inferior infarction: Coronary angiographic findings. Catheterization and Cardiovascular Diagnosis. 1983;9(2):143-151.

18. Kouvaras G, Spyropoulou M, Bacoulas G. The Significance of a Persistent Precordial ST Segment $\geq$ $0.1 \mathrm{mV}$ Depression in Acute Inferior Myocardial Infarction (Coronary Angiographic and Ventriculographic Findings). Angiology. 1986;37(1):57-62.

19. O'Gara PT, Kushner FG, Ascheim DD, et al. 2013 ACCF/AHA Guideline for the Management of ST-Elevation Myocardial Infarction: A Report of the American College of Cardiology Foundation/ American Heart Association Task Force on Practice Guidelines. Journal of the American College of Cardiology. 2013;61(4):e78-e140.

20. Surawicz B, Knilans T. Acute Ischemia : Electrocardiographic Patterns. In: Chou's Electrocardiography in Clinical Practice. 6th edition. Philadelphia: Saunders Elsevier; 2008.

21. Goldberger AL, Goldberger ZD, Shvilkin A. Goldberger's Clinical Electrocardiography: A Simplified Approach. 8th edition. Philadelphia: Saunders Elsevier; 2013.

22. Becker RC, Alpert JS. Electrocardiographic ST segment depression in coronary heart disease. American heart journal, American heart journal, The American Heart Journal, XIV International Congress on Glass - Collected Papers. 1988;115(4):862868.

23. Sianos G, Morel M, Kappetein A, et al. The SYNTAX Score: An angiographic tool grading the complexity of coronary artery disease. EuroIntervention : journal of EuroPCR in collaboration with the Working Group on Interventional Cardiology of the European Society of Cardiology. 2005;1:219-227.

24. Head SJ, Farooq V, Serruys PW, Kappetein AP. The SYNTAX score and its clinical implications. Heart. 2014;100(2):169-177. 